
Olfa Kanoun

\title{
Study of the humidity effect on the electrical impedance of MWCNT epoxy nanocomposites
}

\begin{abstract}
Owing to the excellent physical properties of carbon nanotubes (CNTs), incorporation of CNTs into polymer matrices would make the nanocomposites a potential candidate for smart sensing applications. However, environmental factors such as humidity might influence the nanocomposite properties and performance. In this work, the effect of humidity on the electrical properties of multiwalled CNT (MWCNT) epoxy nanocomposites is investigated by electrochemical impedance spectroscopy in the frequency range from $40 \mathrm{~Hz}$ to $110 \mathrm{MHz}$. The MWCNT epoxy nanocomposites with different MWCNT concentrations up to $1 \%$ wt were fabricated by the direct mixing method and the composites were deposited on the glass epoxy substrate by the stencil printing technique. From the impedance results, it was observed that the overall impedance of the nanocomposites tends to increase with the increase of the humidity due to an increase in tunneling barriers induced by swelling of the polymer layer between the CNT junctions within the nanocomposite matrix. The humidity effect tends to decrease with the increase of MWCNT concentration due to a decrease of the tunneling effect at high MWCNT concentrations. Furthermore, the proposed nanocomposites at a $0.5 \%$ wt concentration show a humidity sensitivity of about $0.006 \%$ relative humidity $(\mathrm{RH})$ and $0.004 \% \mathrm{RH}$ and linear responses with linear correlations $\left(R^{2}\right)$ of 0.98 and 0.93 for $100 \mathrm{~Hz}$ and $1 \mathrm{kHz}$, respectively. Moreover, the sensors were covered with latex polymer to minimize the humidity effects. It was found that with encapsulation of the nanocomposites, the relative impedance change of the nanocomposite is decreased from $3.76 \%$ to $1.15 \%$ at a $0.5 \%$ wt MWCNT concentration.
\end{abstract}

Keywords: carbon nanotubes, epoxy resin, nanocomposites, humidity sensing, encapsulation, electrochemical impedance spectroscopy

\section{Introduction}

Since their discovery of by Iijima in 1991 [1], carbon nanotubes (CNTs) have been used extensively in most fields of science and engineering owing to their excellent mechan-

Acknowledgement: This work was partially supported by the Ministry of National Education of Turkey, which provided Abdulkadir Sanli with a scholarship. The authors gratefully acknowledge Mr. Ammar Al-Hamry for designing the salt solutions for humidity investigations.

Abdulkadir Sanli, Abderrahmane Benchirouf, Christian Müller, Olfa Kanoun, Chair for Measurement and Sensor Technology, Chemnitz University of Technology, Reichenhainer Strasse 70, 09126 Chemnitz, Germany, e-mail: abdulkadir.sanli@s2012.tu-chemnitz.de

https://doi.org/10.1515/9783110558920-002 
ical, thermal and electrical properties [2-5]. In comparison to other fillers, such as nanoparticles or carbon black, CNTs are considered as an effective filler owing to their high aspect ratios $(\approx 100-1000)$, which result in the formation of conductive paths at very low CNT concentrations [6, 7]. Thus, integration of CNTs into a polymer matrix would make the composites a potential candidate for smart sensing applications, including chemical, pressure, gas, flow and mechanical sensors [8-11]. The environmental factors, e. g., humidity, can influence the overall electrical conductivity as well as sensor performance of a polymer nanocomposite for the sensor applications such as strain and pressure sensing, in which the compensation of environmental effects is required. Therefore, it is important to quantify the influence of environmental factors on the electrical conductivity of CNT-based nanocomposites. To reveal the conduction mechanism of the CNT polymer nanocomposites under the humidity effects, several researchers have proposed various explanations [12-17]. For instance, Yoo et al. [13] suggested that resistivity changes are caused by polymer swelling below the percolation threshold, whereas above the percolation threshold the main reason in resistivity change is the charge transfer from water molecules to CNTs. On the other hand, Yu et al. [14] suggested that the change in the conductivity is due to the formation of a weak bond between $\mathrm{H}$ and $\mathrm{C}$ atoms from water and CNTs induced by water uptake. Asrafi et al. [16] have found that a change of humidity from $5 \%$ relative humidity (RH) to $95 \%$ RH can cause an $80 \%$ reduction in conductivity of CNT epoxy nanocomposites due to moisture ingress. However, to the best of our knowledge, there is no study addressing the effects of the CNT concentration and polymer coating on the electrical properties of MWCNT epoxy nanocomposites under humidity effects.

Therefore, this work focuses on the quantitative investigation of the effect of humidity on the electrical conductivity of a free standing and encapsulated MWCNT epoxy nanocomposite by electrochemical impedance spectroscopy for different MWCNT concentrations.

\section{Experimental procedures}

\subsection{Measurement setup for humidity investigations}

Prior to placing the samples inside the salt container, a precise humidity sensor is used to identify the values of humidity and temperature within the salt container. After the sample has been placed inside the container, impedance measurements were taken after ca. $45 \mathrm{~min}$ for the stabilization of the humidity. After that, the impedance data were recorded by utilizing the Agilent 4294A precision impedance analyzer in the frequency range of $40 \mathrm{~Hz}$ to $110 \mathrm{MHz}$. The measurement setup for humidity analysis of MWCNT epoxy nanocomposites is represented in Fig. 1. 

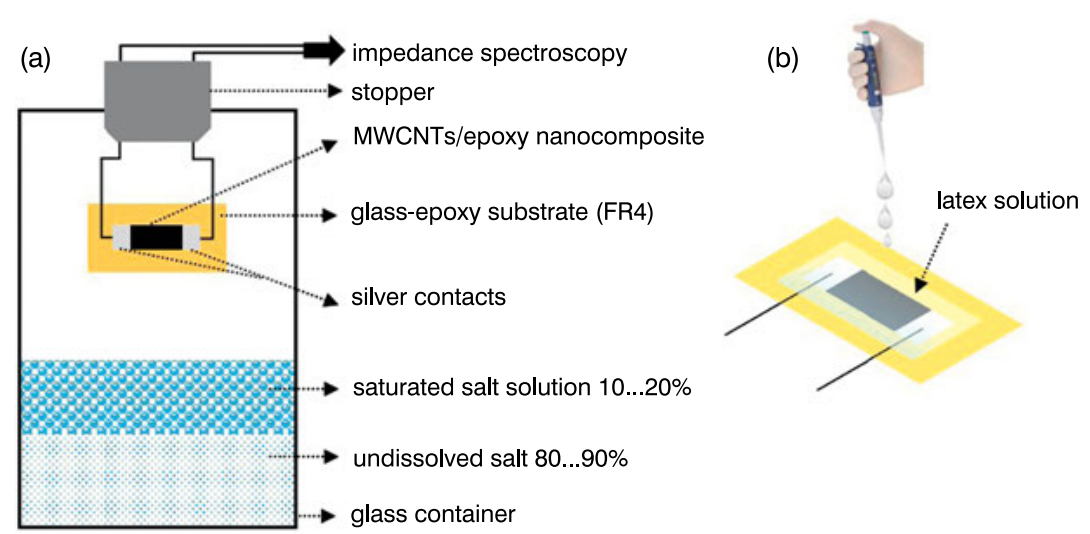

Fig. 1: Schematic illustration of (a) humidity measurement setup for humidity investigations of MWCNT epoxy nanocomposites placed inside a glass container and (b) encapsulation of samples with latex solution.

\section{Results and discussion}

\subsection{Effect of humidity on the impedance response}

It can be seen from both the Nyquist plot and the Bode plot (see Fig. 2) that the impedance of the MWCNT epoxy nanocomposites tend to increase with the increase of RH. The Nyquist plot shows quasi-semi-circle behavior for all concentrations and humidity responses. In order to estimate the complex transport mechanism in MWCNT epoxy nanocomposites under the humidity effect, a 3D R-C model can be proposed to reveal the effect of the MWCNT concentration and the interaction within the nanocom-

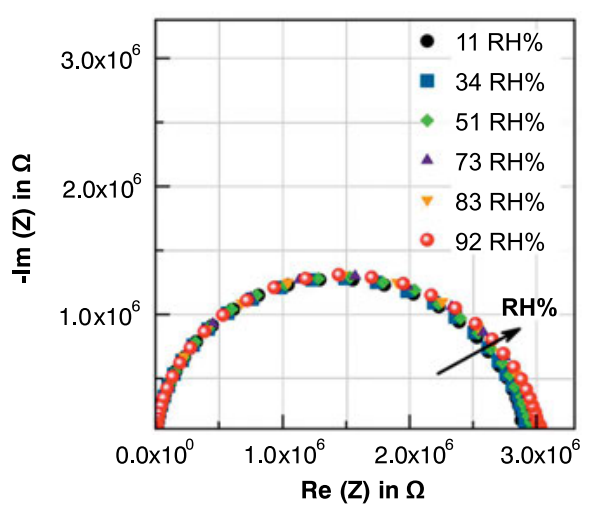

(a)

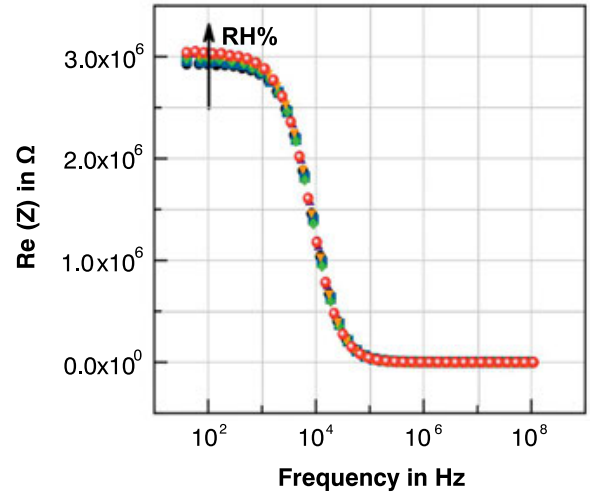

(b)

Fig. 2: (a) Nyquist and (b) Bode plot of non-capsulated MWCNT epoxy nanocomposites under RH. 


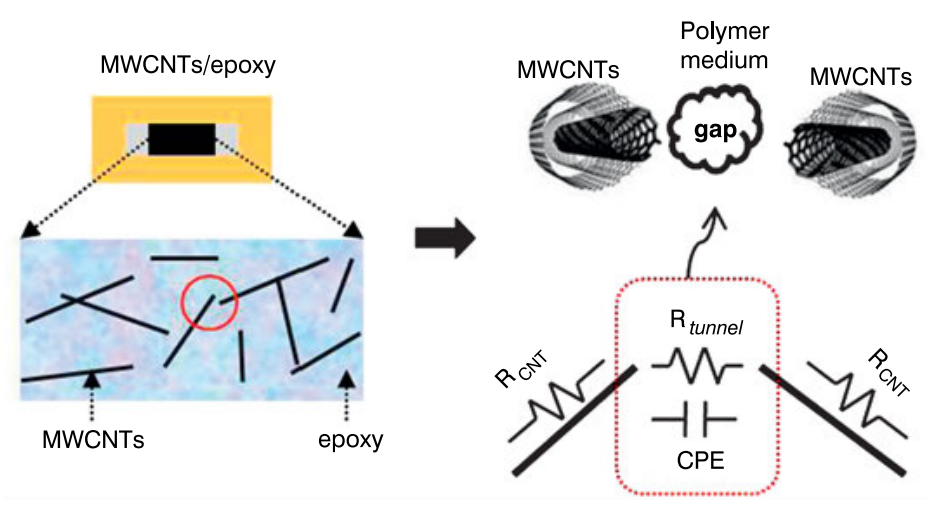

Fig. 3: Illustration of the working mechanism and corresponding equivalent circuit model of an MWCNT epoxy nanocomposite.

posite matrix, shown in Fig. 3. Here, each nanotube (or nanotube agglomerate) has a resistance $\mathrm{R}_{\mathrm{CNTs}}$ and the polymer medium can be modeled as $\mathrm{R}_{\text {tunnel }}$ and constant phase element (CPE), respectively.

\subsubsection{Humidity sensing mechanism}

From the accumulated studies [18-23], the effect of humidity on the CNT polymer nanocomposites can be attributed to the following working mechanisms: (i) the increase of intrinsic resistance of CNTs due to adsorption of water molecules [20], (ii) the charge transfer between the water molecules and CNT modules and the Fermi level that causes the resistance change across CNT-CNT junctions [21, 22] and (iii) the change in inter-tube distance induced by polymer swelling under the humidity [23]. For the case of CNT epoxy nanocomposites, the main reason behind the increase of impedance under humidity is the polymer swelling. Many researchers have suggested that the swelling of the polymer layer between CNT-CNT junctions within the nanocomposite matrix due to humidity causes the increase of the tunneling barriers $[18,23]$. As the polymer expands, the CNTs in the network within the polymer matrix tend to move apart from each other as if the nanocomposite deformed under the tension, which causes the alteration of the complex impedance of the nanocomposites. Thus, the electrical impedance increases under humidity as shown in Fig. 4. Therefore, the effect of intrinsic resistance change of CNTs under humidity can be neglected.

Here, it was also found that the humidity sensitivity of the nanocomposite decreases with increasing the MWCNT concentration due to a decrease in the effect of the tunneling resistance. When the CNT concentration is high, the MWCNTs locate very close within the nanocomposite matrix. Therefore, the inter-tube distances at high concentrations are less effected by the polymer swelling than those near the perco- 


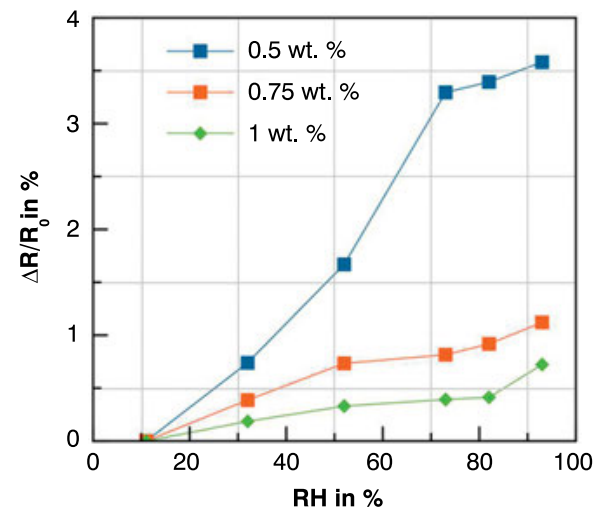

Fig. 4: Relative resistance chance of the MWCNT epoxy nanocomposites under RH for different MWCNT concentrations.

lation threshold [19]. While for the samples of a $0.5 \%$ wt MWCNT concentration the relative resistance change is $3.76 \%$, this value decreases to $0.72 \%$ for $1 \%$ wt MWCNTs. Moreover, as illustrated in Fig. 5, it was seen that the operation frequency has a great effect on the impedance in MWCNT epoxy nanocomposites under RH. It has been observed that the proposed nanocomposites of a $0.5 \mathrm{wt} \%$ MWCNT concentration show humidity sensitivities of about $0.006 \% \mathrm{RH}$ and $0.004 \% \mathrm{RH}$ with linear responses $\left(R^{2}\right)$ of 0.98 and 0.93 for $100 \mathrm{~Hz}$ and $1 \mathrm{kHz}$, respectively. The proposed MWCNT

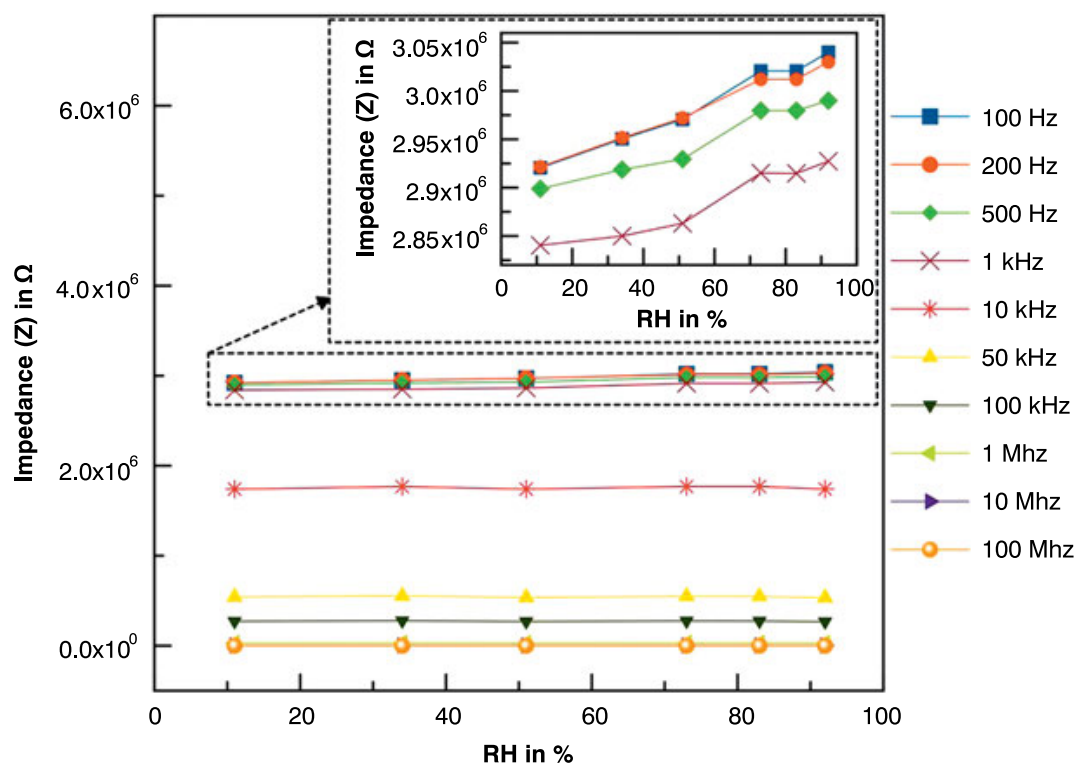

Fig. 5: Impedance response of MWCNT epoxy nanocomposite at a $0.5 \%$ wt MWCNT concentration under RH for different frequencies up to $100 \mathrm{MHz}$. Inset image shows the corresponding impedance response of the MWCNT epoxy nanocomposites up to $1 \mathrm{kHz}$. 
epoxy nanocomposite can be also used as humidity sensor as it was found to be more sensitive than many other nanocomposites [13-24].

\subsection{Effect of the encapsulation on the impedance response}

In order to minimize the effect of the humidity on the electrical properties of MWCNT epoxy nanocomposites, samples were encapsulated with latex polymer (see Fig. 6b). After that, the samples underwent the same humidity measurements again. From Fig. 6, it can be seen that the resistance value of the composites slightly decreases after the encapsulation due to the effect of the latex polymer, which acts as an additional parallel resistance to the composite. By extracting the resistance values of the encapsulated nanocomposites, it can be seen that the latex encapsulation significantly minimizes the effect of the humidity. While the relative resistance change of the nanocomposites for $0.5 \%$ wt decreases from $3.76 \%$ to $1.15 \%$, this value decreases from $0.72 \%$ to $0.52 \%$ for a $1 \%$ wt MWCNT concentration, as shown in Fig. 7 .
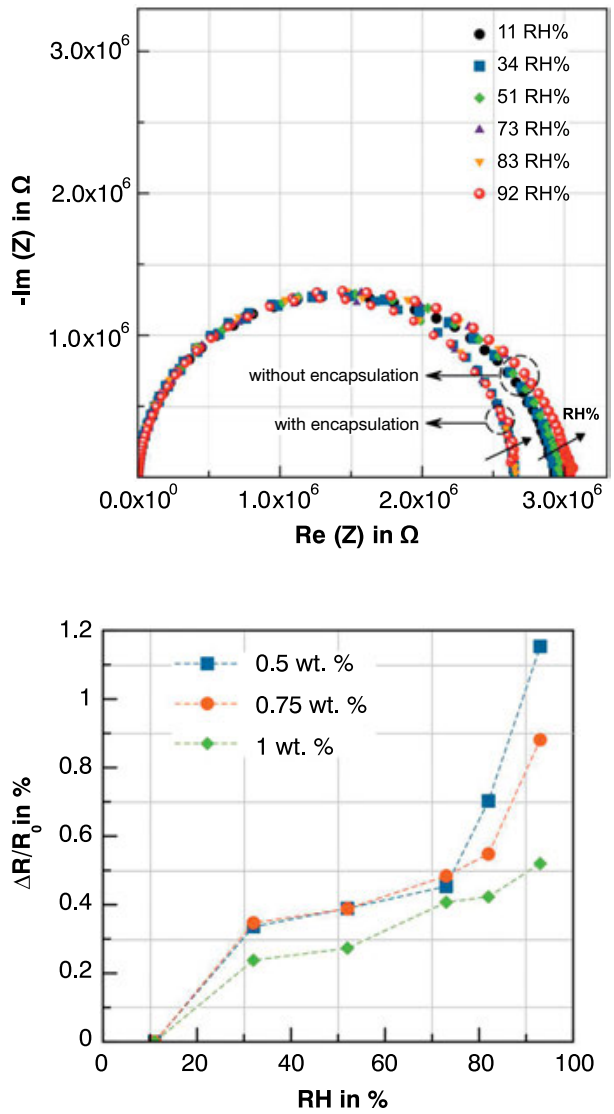

Fig. 6: Nyquist plot of encapsulated and nonencapsulated MWCNT epoxy nanocomposites at a $0.5 \%$ wt MWCNT concentration under up to $92 \% \mathrm{RH}$.

Fig. 7: Relative resistance chance of the latexencapsulated MWCNT epoxy nanocomposites under RH for different MWCNT concentrations. 


\section{Conclusions and outlook}

In this study, we investigated the effect of humidity on the impedance properties of MWCNT epoxy nanocomposites for different MWCNT concentrations. From the impedance results, we found that the impedance of the nanocomposites increases with the increase of the humidity due to an increase in tunneling barriers induced by swelling of the polymer layer. Additionally, the humidity effect was found to decrease with the increase of MWCNT concentration due to the decrease of the tunneling effect at high MWCNT concentrations. Furthermore, the proposed nanocomposites at a 0.5 wt \% MWCNT concentration show a humidity sensitivity of about $0.006 \% \mathrm{RH}$ and a linear response $\left(R^{2}\right)$ of 0.98 . Moreover, by encapsulation of the nanocomposites the relative impedance change of the nanocomposite decreases from $3.76 \%$ to $1.15 \%$ at a $0.5 \%$ wt MWCNT concentration. For the future work, it would be interesting to explore the effect of the different encapsulation methods on the electrical and piezoresistive properties of the MWCNT epoxy nanocomposites.

\section{Bibliography}

[1] S. Bianco, C. F. Pirri, M. Quaglio, P. Ferrario, and R. Castagna, "Nanocomposites based on elastomeric matrix filled with carbon nanotubes for biological applications", INTECH Open Access Publisher.

[2] Z. Yao, C. L. Kane, and C. Dekker, "High-field electrical transport in single-wall carbon nanotubes”, Physical Review Letters, vol. 84, no. 13, p. 2941.

[3] P. R. Bandaru, "Electrical properties and applications of carbon nanotube structures", Journal of Nanoscience and Nanotechnology, vol. 7, no. 4-5, pp. 1239-1267.

[4] M. J. Treacy, T. W. Ebbesen, and J. M. Gibson, "Exceptionally high Young's modulus observed for individual carbon nanotubes".

[5] M. F. Yu, B. S. Files, S. Arepalli, and R. S. Ruoff, "Tensile loading of ropes of single wall carbon nanotubes and their mechanical properties”, Physical Review Letters, vol. 84, no. 24, p. 5552.

[6] J. K. W. Sandler, S. Pegel, M. Cadek, F. Gojny, M. Van Es, J. Lohmar, and M. S. P. Shaffer, "A comparative study of melt spun polyamide-12 fibres reinforced with carbon nanotubes and nanofibres", Polymer, vol. 45, no. 6, pp. 2001-2015.

[7] D. S. McLachlan, C. Chiteme, C. Park, K. E. Wise, S. E. Lowther, P. T. Lillehei, and J. S. Harrison, "AC and DC percolative conductivity of single wall carbon nanotube polymer composites", Journal of Polymer Science Part B, Polymer Physics, vol. 43, no. 22, pp. 3273-3287.

[8] O. Kanoun, C. Müller, A. Benchirouf, A. Sanli, T. N. Dinh, A. Al-Hamry, and A. Bouhamed, "Flexible carbon nanotube films for high performance strain sensors", Sensors, vol. 14, no. 6, pp. 10042-10071.

[9] A. Benchirouf, S. Palaniyappan, R. Ramalingame, P. Raghunandan, T. Jagemann, C. Müller, and O. Kanoun, "Electrical properties of multi-walled carbon nanotubes/PEDOT: PSS nanocomposites thin films under temperature and humidity effects", Sensors and Actuators B, Chemical, vol. 224, pp.344-350. 
[10] O. Kanoun, C. Müller, A. Benchirouf, A. Sanli, A. Bouhamed, A. Al-Hamry, and L. Bu, "Potential of flexible carbon nanotube films for high performance strain and pressure sensors", Nanotechnology for Optics and Sensors, One Central (OCP).

[11] A.Sanli, C. Müller, O. Kanoun, C. Elibol, and M. F. X. Wagner, "Piezoresistive characterization of multi-walled carbon nanotube-epoxy based flexible strain sensitive films by impedance spectroscopy", Composites Science and Technology, vol. 122, pp.18-26.

[12] T. Fei, K. Jiang, F. Jiang, R. Mu, and t. Zhang, "Humidity switching properties of sensors based on multiwalled carbon nanotubes/polyvinyl alcohol composite films", Journal of Applied Polymer Science, vol.131, no. 1.

[13] K. P. Yoo, L. T. Lim, N. K. Min, M. J. Lee, C. J. Lee, and C. W. Park, "Novel resistive-type humidity sensor based on multiwall carbon nanotube/polyimide composite films", Sensors and Actuators B, Chemical, vol. 145, no. 1, pp.120-125.

[14] H. Yu, T. Cao, L. Zhou, E. Gu, D. Yu, and D. Jiang, "Layer-by-layer assembly and humidity sensitive behavior of poly (ethyleneimine)/multiwall carbon nanotube composite films", Sensors and Actuators B, Chemical, vol. 119, no. 2, pp. 512-515.

[15] P. G. Su and C. S. Wang, "In situ synthesized composite thin films of MWCNTs/PMMA doped with $\mathrm{KOH}$ as a resistive humidity sensor", Sensors and Actuators B, Chemical, vol. 124, no. 2, pp. 303-308.

[16] Q.Y. Tang, Y. C. Chan, and K. Zhang, "Fast response resistive humidity sensitivity of polyimide/multiwall carbon nanotube composite films", Sensors and Actuators B, Chemical, vol. 152, no. 1, pp. 99-106.

[17] B. Ashrafi, D. Marchand, Y. Martinez-Rubi, M. B. Jakubinek, B. Simard, and A. Johnston, "Effect of Humidity on Electrical conductivity of Carbon Nanotube-Modified Epoxy".

[18] L. Liu, X. Ye, K. Wu, Z. Zhou, D. Lee, and T. Cui, "Humidity sensitivity of carbon nanotube and poly (dimethyldiallylammonium chloride) composite films”, IEEE Sensors Journal, vol. 9, no. 10, pp. 1308-1314.

[19] H. Lei, W. G. Pitt, L. K. McGrath, and C. K. Ho, "Modeling carbon black/polymer composite sensors", Sensors and Actuators B, Chemical, vol. 125, no. 2, pp. 396-407.

[20] A. Zahab, L. Spina, P. Poncharal, and C. Marliere, "Water-vapor effect on the electrical conductivity of a single-walled carbon nanotube mat", Physical Review B, vol. 62, no. 15, p. 10000.

[21] J. Li, Y. Lu, Q. Ye, M. Cinke, J. Han, and M. Meyyappan, "Carbon nanotube sensors for gas and organic vapor detection”, Nano Letters, vol. 3, no. 7, pp. 929-933.

[22] J. Zhao, A. Buldum, J. Han, and J. P. Lu, "Gas molecule adsorption in carbon nanotubes and nanotube bundles", Nanotechnology, vol.13, no. 2, p. 195.

[23] D. Sung, S. Hong, Y. Kim, N. Park, S. Kim, S. L. Maeng, and K. C Kim, "Ab initio study of the effect of water adsorption on the carbon nanotube field-effect transistor", Applied Physics Letters, vol. 89, no. 24, p. 243110.

[24] L. Liu, X. Ye, K. Wu, R. Han, Z. Zhou, and T. Cui, "Humidity sensitivity of multi-walled carbon nanotube networks deposited by dielectrophoresis", Sensors, vol. 9, no. 3, pp.1714-1721, 2009. 